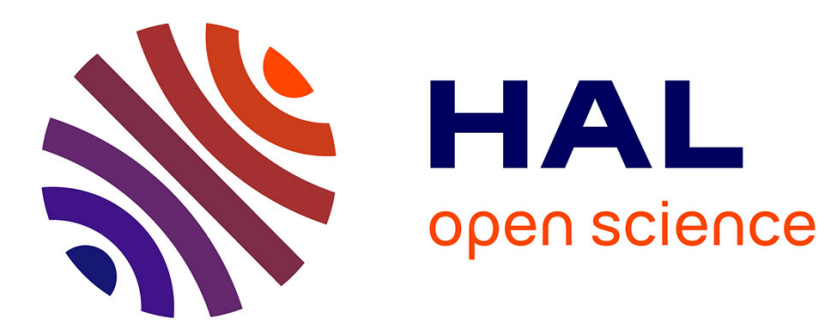

\title{
Cointegration in frequency domain
}

\author{
Daniel Levy
}

\section{To cite this version:}

Daniel Levy. Cointegration in frequency domain. Journal of Time Series Analysis, 2002, 23 (3), pp.333-339. 10.1111/1467-9892.00267 . hal-02385599

\section{HAL Id: hal-02385599 \\ https://hal.science/hal-02385599}

Submitted on 28 Nov 2019

HAL is a multi-disciplinary open access archive for the deposit and dissemination of scientific research documents, whether they are published or not. The documents may come from teaching and research institutions in France or abroad, or from public or private research centers.
L'archive ouverte pluridisciplinaire HAL, est destinée au dépôt et à la diffusion de documents scientifiques de niveau recherche, publiés ou non, émanant des établissements d'enseignement et de recherche français ou étrangers, des laboratoires publics ou privés. 


\title{
Cointegration in Frequency Domain*
}

\author{
Daniel Levy \\ Department of Economics \\ Bar-Ilan University \\ Ramat-Gan 52900 \\ ISRAEL
}

Tel: (972)-3-531-8331

Fax: (972)-3-535-3180

LEVYDA@MAIL.BIU.AC.IL

and
Department of Economics
Emory University
Atlanta, GA 30322
USA

Tel: (404) 727-2941

Fax: (404) 727-4639

ECONDL@EMORY.EDU

August 18, 2000

Revised: April, 2002

Keywords: common stochastic trend; cointegration: frequency domain anlysis; cross-spectrum; zero-frequency.

\footnotetext{
* The manuscript was completed when I was a Visiting Associate Professor at Bar-Ilan University. I am grateful to the anonymous referee for important suggestions, which led to the generalization of the results reported in this paper, and to Andy Young for comments. All errors are mine.
} 


\title{
Cointegration in Frequency Domain*
}

\begin{abstract}
Existence of a cointegration relationship between two time series in the time domain imposes restrictions on the series zero-frequency behaviour in terms of their squared coherence, phase, and gain, in the frequency domain. I derive these restrictions by studying cross-spectral properties of a cointegrated bivariate system. Specifically, I demonstrate that if two difference stationary series, $X_{t}$ and $Y_{t}$, are cointegrated with a cointegrating vector $\left[\begin{array}{ll}1 & b\end{array}\right]$ and thus share a common stochastic trend, then at the zero frequency, the squared coherence of $(1-L) X_{t}$ and $(1-L) Y_{t}$ will equal one, their phase will equal zero, and their gain will equal $|b|$.
\end{abstract}




\section{Introduction}

Since the introduction of cointegration and common trend analysis in econometrics and statistics by Engle and Granger (1987) and Stock and Watson (1988), integration and cointegration tests have by now become an essential part of the applied econometricians' and macroeconomists' standard tool kit. These tests are routinely applied to economic time series because the notion of cointegration has a natural economic interpretation: existence of a cointegration relationship between two variables indicates that the series "move together" in the long run, and so they share a common stochastic trend, although in the short run the series may diverge from each other. Since many economic theories make these kinds of long-run and short-run differential predictions about economic time series co-movements, many economic models (and particularly macroeconomic models) lend themselves naturally to cointegration testing (Engle and Grange, 1987).

The cointegtation property is a long-run property, and therefore in frequency domain it refers to the zero-frequency relationship of the time series. Therefore, there is a frequency-domain equivalent of the time-domain cointegration property. Specifically, existence of a cointegration relationship between two time series in the time domain imposes restrictions on the series zero-frequency behavior in terms of their cross spectral measures in the frequency domain. The purpose of this paper is to use a bivariate setting to derive these frequency-domain restrictions in terms of the time series' squared coherence, phase and gain, which are the measures practitioners typically consider when studying cross spectral properties of time series.

Squared coherence is analogous to the square of the correlation coefficient and measures the degree to which one series can be represented as a linear function of the other. Phase measures the phase difference or the timing (i.e., lead or lag) between the 
frequency components of the two series. Gain indicates how much the spectrum of one series has been amplified to approximate the corresponding frequency component of the other. It is essentially the regression coefficient of one series on another at frequency $\omega$. Thus, the squared coherence, phase and gain are frequency-domain equivalents of the correlation coefficient, time-delay (lag), and regression coefficient, respectively, and, therefore, they have a natural interpretation in terms of the standard time domain regression analysis.

The paper proceeds as follows: I derive cross spectral properties of a cointegrated bivariate system by beginning with two non-stationary time series that are cointegrated with a cointegration vector $\left[\begin{array}{ll}1 & b\end{array}\right]$, and using standard Fourier Transform methods and matrix algebra, I derive frequency domain properties of the series' comovement in terms of their squared coherence, phase and gain. Specifically, I show that the squared coherence between such series, after differencing, will equal one, their phase will equal zero, while their gain will equal $|b|$. The paper ends with a brief conclusion in Section 3.

\section{Cross-Spectral Properties of a Cointegrated Bivariate System}

Let the time series of $X_{t}$ and $Y_{t}$ be difference stationary. Thus, let $X_{t} \sim \mathrm{I}(1)$ and $Y_{t} \sim \mathrm{I}(1)$, so that they can be written as

$$
X_{t}=X_{t-1}+u_{t}
$$

and

$$
Y_{t}=Y_{t-1}+v_{t}
$$


respectively, where $u_{t} \sim \mathrm{I}(0)$, and $v_{t} \sim \mathrm{I}(0)$. Moreover, let us assume that $X_{t}$ and $Y_{t}$ are cointegrated with the cointegrtaion vector $\left[\begin{array}{ll}1 & b\end{array}\right]$, so that they satisfy

$$
Y_{t}=b X_{t}+\mu_{t}
$$

where $\mu \sim I(0)$. Then, $X_{t}$ and $Y_{t}$ processes share a common stochastic trend and, therefore, can be written in a matrix notation

$$
\left|\begin{array}{l}
X_{t} \\
Y_{t}
\end{array}\right|=\left[\begin{array}{l}
T_{t}+x_{t} \\
b T_{t}+y_{t}
\end{array}\right]
$$

where $T_{t}$ is the common stochastic trend with the property

$(1-L) T_{t}=z_{t}, z_{t} \sim \operatorname{iid}\left(0, \sigma_{z}^{2}\right)$ is a white noise process, $x_{t} \sim \mathrm{I}(0)$, and $y_{t} \sim \mathrm{I}(0)$.

Applying difference operator $\Delta=(1-L)$ to (1) yields a bivariate stationary process

$$
\left[\begin{array}{l}
(1-L) X_{t} \\
(1-L) Y_{t}
\end{array}\right]=\left[\begin{array}{l}
z_{t}+(1-L) x_{t} \\
b z_{t}+(1-L) y_{t}
\end{array}\right]
$$

with the special matrix

$$
\mathbf{f}(\omega)=\left[\begin{array}{ll}
f_{\Delta X} & f_{\Delta X \Delta Y} \\
f_{\Delta Y \Delta X} & f_{\Delta Y}
\end{array}\right]
$$

The diagonal elements of the $\mathbf{f}(\omega)$ matrix are the spectral density functions of $(1-L) X_{t}$ and $(1-L) Y_{t}$, defined by:

$$
f_{\Delta X}=\frac{1}{2 \pi} \int_{-\infty}^{\infty} \gamma_{\Delta X}(\tau) e^{-i \tau \omega} d \tau
$$




$$
f_{\Delta Y}=\frac{1}{2 \pi} \int_{-\infty}^{\infty} \gamma_{\Delta Y}(\tau) e^{-i \tau \omega} d \tau
$$

where $\gamma_{\Delta X}(\tau)$ and $\gamma_{\Delta Y}(\tau)$ are the autocovariance functions of $(1-L) X_{t}$ and $(1-L) Y_{t}$, defined by

$$
\begin{aligned}
& \gamma_{\Delta X}(\tau)=E\left[\left(\Delta X_{t+\tau}-\mu_{\Delta X}\right)\left(\Delta X_{t}-\mu_{\Delta X}\right)\right] \\
& \gamma_{\Delta y}(\tau)=E\left[\left(\Delta Y_{t+\tau}-\mu_{\Delta Y}\right)\left(\Delta Y_{t}-\mu_{\Delta Y}\right)\right],
\end{aligned}
$$

respectively, where $\mu_{\Delta X}$ and $\mu_{\Delta Y}$ denote the means of $(1-L) X_{t}$ and $(1-L) Y_{t}$, respectively. The off-diagonal elements of the $\mathbf{f}(\omega)$ matrix are the cross-spectral density functions of $(1-L) X_{t}$ and $(1-L) Y_{t}$, defined by

$$
\begin{aligned}
& f_{\Delta X \Delta Y}=\frac{1}{2 \pi} \int_{-\infty}^{\infty} \gamma_{\Delta X \triangle Y}(\tau) e^{-i \tau \omega} d \tau \\
& f_{\Delta Y \Delta X}=\frac{1}{2 \pi} \int_{-\infty}^{\infty} \gamma_{\Delta Y X X}(\tau) e^{-i \tau \omega} d \tau
\end{aligned}
$$

where $\gamma_{\triangle X \Delta Y}(\tau)$ and $\gamma_{\triangle Y \Delta X}(\tau)$ are the crosscovariance functions of $(1-L) X_{t}$ and $(1-L) Y_{t}$, and $(1-L) Y_{t}$ and $(1-L) X_{t}$, defined by

$$
\begin{aligned}
& \gamma_{\Delta X \Delta Y}(\tau)=E\left[\left(\Delta X_{t+\tau}-\mu_{\Delta X}\right)\left(\Delta Y_{t}-\mu_{\Delta Y}\right)\right] \\
& \gamma_{\Delta Y \Delta X}(\tau)=E\left[\left(\Delta Y_{t+\tau}-\mu_{\Delta Y}\right)\left(\Delta X_{t}-\mu_{\Delta X}\right)\right],
\end{aligned}
$$

respectively. 
To compute the elements of the $\mathbf{f}(\omega)$ matrix, first compute the autocovariancecrosscovariance matrix of (2), which is given by:

$$
\begin{aligned}
\gamma(\tau) & =\left[\begin{array}{l}
\gamma_{\Delta X}(\tau) \gamma_{\Delta X \Delta Y}(\tau) \\
\gamma_{\Delta Y \Delta X}(\tau) \gamma_{\Delta Y}(\tau)
\end{array}\right] \\
& =E\left[\begin{array}{l}
z_{t+\tau}+\Delta x_{t+\tau} \\
b z_{t+\tau}+\Delta y_{t+\tau}
\end{array}\right]\left[\begin{array}{l}
\left.z_{t}+\Delta x_{t} b z_{t}+\Delta y_{t}\right] \\
\end{array}=E\left[\begin{array}{ll}
\left(z_{t+\tau}+\Delta x_{t+\tau}\right)\left(z_{t}+\Delta x_{t}\right) & \left(z_{t+\tau}+\Delta x_{t+\tau}\right)\left(b z_{t}+\Delta y_{t}\right) \\
\left(b z_{t+\tau}+\Delta y_{t+\tau}\right)\left(z_{t}+\Delta x_{t}\right)\left(b z_{t+\tau}+\Delta y_{t+\tau}\right)\left(b z_{t}+\Delta y_{t}\right)
\end{array}\right]\right. \\
& =\left[\begin{array}{ll}
\gamma_{z}(\tau)+\gamma_{1}(\tau)+\gamma_{z 1}(\tau)+\gamma_{1 z}(\tau) & b \gamma_{z}(\tau)+b \gamma_{1 z}(\tau)+\gamma_{z 2}(\tau)+\gamma_{12}(\tau) \\
b \gamma_{z}(\tau)+\gamma_{2 z}(\tau)+b \gamma_{z 1}(\tau)+\gamma_{21}(\tau) & \left.b^{2} \gamma_{z}(\tau)+\gamma_{2} \tau\right)+b \gamma_{z 2}(\tau)+b \gamma_{2 z}(\tau)
\end{array}\right],
\end{aligned}
$$

where subscripts 1 and 2 denote $\Delta x$ and $\Delta y$, respectively, for notational simplicity, the diagonal elements of the last matrix in (8) are the autocovariance functions, $\gamma_{\Delta X}(\tau)$ and $\gamma_{\Delta Y}(\tau)$, and the off-diagonal elements are the crosscovariance functions $\gamma_{\Delta X \Delta Y}(\tau)$ and $\gamma_{\Delta Y \Delta X}(\tau)$, respectively, as defined in (5a)-5(b) and 7(a)-7(b).

Applying the Fourier Transform to both sides of equation (8), multiplying through by $\frac{1}{2 \pi}$, and using the spectrum and cross-spectrum definitions provided by (4a)-4(b) and 6(a)-6(b), we get the special matrix

$$
\mathbf{f}(\omega)=\left[\begin{array}{lr}
f_{z}(\omega)+f_{1}(\omega)+f_{z 1}(\omega)+f_{1 z}(\omega) & b f_{z}(\omega)+b f_{1 z}(\omega)+f_{z 2}(\omega)+f_{12}(\omega) \\
b f_{z}(\omega)+f_{2 z}(\omega)+b f_{z 1}(\omega)+f_{21}(\omega) & b^{2} f_{z}(\omega)+f_{2}(\omega)+b f_{z 2}(\omega)+b f_{2 z}(\omega)
\end{array}\right]
$$

which can be rewritten as

$$
\mathbf{f}(\omega)=\left[\begin{array}{ll}
f_{z}(\omega) & b f_{z}(\omega) \\
b f_{z}(\omega) & b^{2} f_{z}(\omega)
\end{array}\right]+\left[\begin{array}{ll}
f_{1}(\omega)+f_{z 1}(\omega)+f_{1 z}(\omega) & b f_{1 z}(\omega)+f_{z 2}(\omega)+f_{12}(\omega) \\
f_{2 z}(\omega)+b f_{z 1}(\omega)+b f_{21}(\omega) & f_{2}(\omega)+b f_{z 2}(\omega)+b f_{2 z}(\omega)
\end{array}\right](10)
$$


The cross spectrum in (10) can be written in Cartesian form because the spectral matrix $\mathbf{f}(\omega)$ is in general a complex valued function. Thus, for example, we can write

$$
f_{m n}(\omega)=c_{m n}(\omega)-i q_{m n}(\omega),
$$

where $c_{m n}(\omega)$ denotes the cospectral density function of $m$ and $n$, and $q_{m n}(\omega)$ denotes the quadrature spectral density function of $m$ and $n$. Therefore, using Priestley's (1981, p. 668, Equation 9.1.53) result that $f_{m n}(\omega)=\overline{f_{n m}(\omega)},(10)$ can be rewritten as

$$
\mathbf{f}(\omega)=\left[\begin{array}{ll}
f_{z}(\omega) & b f_{z}(\omega) \\
b f_{z}(\omega) & b^{2} f_{z}(\omega)
\end{array}\right]+\left[\begin{array}{ll}
f_{1}(\omega)+f_{z 1}(\omega)+\overline{f_{z 1}(\omega)} & b f_{1 z}(\omega)+f_{z 2}(\omega)+f_{12}(\omega) \\
f_{2 z}(\omega)+b f_{z 1}(\omega)+f_{21}(\omega) & f_{2}(\omega)+b f_{z 2}(\omega)+b \overline{f_{z 2}(\omega)}
\end{array}\right]
$$

where bar denotes a complex conjugate. Combining (12) with Cartesian representation of $f_{1}(\omega)$ and $f_{z 2}(\omega)$,

$$
f_{z 1}(\omega)=c_{z 1}(\omega)-i q_{z 1}(\omega)
$$

and

$$
f_{z 2}(\omega)=c_{z 2}(\omega)-i q_{z 2}(\omega)
$$

yields

$$
\mathbf{f}(\omega)=\left[\begin{array}{ll}
f_{z}(\omega) & b f_{z}(\omega) \\
b f_{z}(\omega) & b^{2} f_{z}(\omega)
\end{array}\right]+\left[\begin{array}{cc}
f_{1}(\omega)+2 c_{z 1}(\omega) & b f_{1 z}(\omega)+f_{z 2}(\omega)+f_{12}(\omega) \\
f_{2 z}(\omega)+b f_{z 1}(\omega)+f_{21}(\omega) & f_{2}(\omega)+2 b c_{z 2}(\omega)
\end{array}\right]
$$

Now, consider the value of the spectral matrix $\mathbf{f}(\omega)$ at frequency $\omega=0$, which using (3) and (14) can be written as 


$$
\begin{aligned}
\mathbf{f}(0) & =\left[\begin{array}{ll}
f_{\Delta X}(0) & f_{\Delta X \Delta Y}(0) \\
f_{\Delta Y \Delta X}(0) & f_{\Delta Y}(0)
\end{array}\right] \\
& =\left[\begin{array}{ll}
f_{z}(0) & b f_{z}(0) \\
b f_{z}(0) & b^{2} f_{z}(0)
\end{array}\right]+\left[\begin{array}{ll}
f_{1}(0)+2 c_{z 1}(0) & b f_{1 z}(\omega)+f_{z 2}(0)+f_{12}(0) \\
f_{2 z}(0)+b f_{z 1}(0)+f_{21}(0) & f_{2}(0)+2 b c_{z 2}(0)
\end{array}\right] .
\end{aligned}
$$

Recall that $z_{t}$ is a white noise process, and therefore, its theoretical spectrum is flat and equals $f_{z}(\omega)=\sigma_{z}^{2} / 2 \pi$ for all frequencies $-\pi \leq \omega \leq \pi$. In addition, $\Delta x$ and $\Delta y$ are $\mathrm{I}(-1)$, and therefore their zero-frequency spectral density, cross spectral density and cospectral density functions equal zero. Thus, every element of the second matrix of the right hand side of (15) vanishes, and therefore the spectral matrix, evaluated at frequency $\omega=0$, becomes

$$
\begin{aligned}
\mathbf{f}(0) & =\left[\begin{array}{ll}
f_{\Delta X}(0) & f_{\Delta X \Delta Y}(0) \\
f_{\Delta Y \Delta X}(0) & f_{\Delta Y}(0)
\end{array}\right] \\
& =\left[\begin{array}{ll}
\frac{\sigma_{z}^{2}}{2 \pi} & \frac{b \sigma_{z}^{2}}{2 \pi} \\
\frac{b \sigma_{z}^{2}}{2 \pi} & \frac{b^{2} \sigma_{z}^{2}}{2 \pi}
\end{array}\right] .
\end{aligned}
$$

To see the implications of this result for the behavior of the theoretical squared coherence, phase and gain, recall from polar representation of $\mathbf{f}(\omega)$ that

$$
\begin{aligned}
& K_{\Delta X \Delta Y}^{2}(\omega)=\left|f_{\Delta X \Delta Y}(\omega)\right|^{2}\left[f_{\Delta X}(\omega) f_{\Delta Y}(\omega)\right]^{-1} \\
& \phi_{\Delta X \Delta Y}(\omega)=\arctan \left\{-\frac{\operatorname{Im}\left[f_{\Delta X \Delta Y}(\omega)\right]}{\operatorname{Re}\left[f_{\Delta X \Delta Y}(\omega)\right]},\right.
\end{aligned}
$$

and 


$$
\Gamma_{\Delta X \Delta Y}(\omega)=\mid f_{\Delta X \Delta Y}(\omega)\left[f_{\Delta X}(\omega)\right]^{-1},
$$

where $\operatorname{Im}\left[f_{\Delta X \Delta Y}(\omega)\right]$ and $\operatorname{Re}\left[f_{\Delta X \Delta Y}(\omega)\right]$ denote the imaginary and real parts of $\left[f_{\Delta X A \Delta}(\omega)\right]$, and $K_{\Delta X \Delta Y}^{2}(\omega), \phi_{\Delta X \Delta Y}(\omega)$, and $\Gamma_{\Delta X \Delta Y}(\omega)$ denote the squared coherence, phase, and the gain of $(1-L) X_{t}$ and $(1-L) Y_{t}$, respectively (Jenkins and Watts, 1968). Then, using the matrix (16) along with the definitions of squared coherence, phase, and gain provided in (17), (18) and (19), we get that at the zero frequency the following equalities hold.

For the squared coherence of $(1-L) X_{t}$ and $(1-L) Y_{t},(16)$ and (17) imply that at frequency $\omega=0$,

$$
K_{\Delta X \Delta Y}^{2}(0)=\left|\frac{b \sigma_{z}^{2}}{2 \pi}\right|\left(\frac{\sigma_{z}^{2}}{2 \pi} \frac{b^{2} \sigma_{z}^{2}}{2 \pi}\right)^{-1}=1
$$

To determine the phase of $(1-L) X_{t}$ and $(1-L) Y_{t}$, note that from the Cartesian representation of $f_{\Delta X \Delta Y}(\omega)$, we can write

$$
f_{\Delta X \Delta Y}(\omega)=c_{\Delta X \Delta Y}(\omega)-i q_{\Delta X \Delta Y} \omega
$$

However, from (16) we know that at zero frequency

$$
f_{\Delta X \Delta Y}(0)=\frac{b \sigma_{z}^{2}}{2 \pi}
$$

Rewrite (21) for $\omega=0$,

$$
f_{\Delta X \Delta Y}(0)=c_{\Delta X \Delta Y}(0)-i q_{\Delta X \Delta Y}(0)
$$


and compare the resulting equation (23) to equation (22). The equality of the two equations requires that their right hand sides be equal. However, we know that for a complex number to equal a real number, it is necessary that the imaginary part of the complex number be zero. In other words, it is necessary that the imaginary part of the complex number be zero. In other words, for equality of (22) and (23), it is necessary that at the frequency $\omega=0$, the cospectrum of $(1-L) X_{t}$ and $(1-L) Y_{t}$ satisfy

$$
\begin{aligned}
c_{\Delta X \Delta Y}(0) & =\operatorname{Re}\left[f_{\Delta X \Delta Y}(0)\right] \\
= & \frac{b \sigma_{z}^{2}}{2 \pi}
\end{aligned}
$$

and the quadrature spectrum of $(1-L) X_{t}$ and $(1-L) Y_{t}$ satisfy

$$
\begin{aligned}
q_{\Delta X \Delta Y}(0) & =\operatorname{Im}\left[f_{\Delta X \Delta Y}(0)\right] \\
& =0
\end{aligned}
$$

Substituting (24) and (25) into the definitions of phase (18) for the frequency $\omega=0$, we find that

$$
\begin{aligned}
\phi_{\Delta X \Delta Y}(0) & =\arctan \left\{-\frac{\operatorname{Im}\left[f_{\Delta X \Delta Y}(0)\right]}{\operatorname{Re}\left[f_{\Delta X \Delta Y}(0)\right]}\right\} \\
& =\arctan (0) \\
& =|0|
\end{aligned}
$$

Finally, to determine the gain of $(1-L) X_{t}$ and $(1-L) Y_{t}$, we need to combine (16) and (19) and evaluate the result for the frequency $\omega=0$. This yields 


$$
\begin{aligned}
\Gamma_{\Delta X \Delta Y}(0) & =\left|\frac{b \sigma_{z}^{2}}{2 \pi}\right|\left[\frac{\sigma_{z}^{2}}{2 \pi}\right]^{-1} \\
& =|b|,
\end{aligned}
$$

where $b$ is the coefficient that measures the extent of the long run relationship between $X_{t}$ and $Y_{t}$. That is, $b$ is the coefficient in the cointegration relationship, $Y_{t}=b X_{t}+\mu_{t}$, where $\mu_{t} \sim I(0)$.

Equations (20), (26) and (27) establish the main results of this paper: if two difference stationary series, $X_{t}$ and $Y_{t}$, are cointegrated with the cointegtrating vector $\left[\begin{array}{ll}1 & b\end{array}\right]$, then the zero frequency squared coherence, phase, and gain of $(1-L) X_{t}$ and $(1-L) Y_{t}$ will equal one, zero, and $|b|$, respectively. This is a generalization of Levy (2000), which only focuses on the behaviour of squared coherence and gain, and only for the case $b=-1$.

\section{Conclusion}

The contigration property is a long-run property, and therefore in the frequency domain, it refers to the zero-frequency relationship of the time series. Therefore there is a frequency-domain equivalent of the time-domain cointegration property: existence of a cointegration relationship between two time series in the time domain, imposes restrictions on the series zero-frequency behavior in terms of their squared coherence, phase, and gain in the frequency domain. In this paper, I derive these frequencydomain restrictions in a bivariate setting. Specifically, I demonstrate that if two difference stationary series, $X_{t}$ and $Y_{t}$, are cointegrated with the cointegrating vector $\left[\begin{array}{ll}1 & b\end{array}\right]$, then the zero frequency squared coherence, phase, and gain of $(1-L) X_{t}$ and $(1-L) Y_{t}$ will equal one, zero, and $|b|$, respectively. 
It is well known that the standard time series cointegration tests have a low power. The results derived in this paper suggest that it may be useful to test for cointegration in the frequency domain. Future work should examine limiting null distributions and finite sample properties of such tests, in order to assess their practical usefulness. 


\section{References}

Engle, R.E. and Granger, C.J.W. (1987) Cointegration and error correction: representation, estimation, and testing, Econometrica 55, 251-276.

Jenkins, G.M. and Watts, D.G. (1968) Spectral Analysis and its Applications. San Francisco: Holden Day.

Levy, D. (2000) Investment-saving comovement and capital mobility: evidence from century-long US time series, Review of Economic Dynamics 3, 100-136.

Priestley, M.B. (1981) Spectral Analysis and Time Series. New York: Academic Press.

Stock, J.M. and Watson, M.W. (1988) Testing for common trends, Journal of American Statistical Association 83, 1097-1107. 